\title{
Evaluation of Red Cell Distribution Width and Platelet Indices in Children with Chronic Heart Disease
}

\author{
Hanan F. Hussein'1, Eglal H. Al-Gohary², Amal G. Mohamed², Amal A. A. Abd El-Salam³ \\ ${ }^{1}$ Al-Zahraa Hospital, Tripoli, Libya \\ ${ }^{2}$ Pediatrics Department, Faculty of Medicine for Girls, Al-Azar University, Cairo, Egypt \\ ${ }^{3}$ National Heart Institute, Cairo, Egypt \\ Email: hanan.farg198610@gmail.com
}

How to cite this paper: Hussein, H.F., Al-Gohary, E.H., Mohamed, A.G. and ElSalam, A.A.A.A. (2021) Evaluation of Red Cell Distribution Width and Platelet Indices in Children with Chronic Heart Disease. Open Journal of Pediatrics, 11, 78-99. https://doi.org/10.4236/ojped.2021.111008

Received: December 29, 2020

Accepted: March 6, 2021

Published: March 9, 2021

Copyright $\odot 2021$ by author(s) and Scientific Research Publishing Inc. This work is licensed under the Creative Commons Attribution International License (CC BY 4.0).

http://creativecommons.org/licenses/by/4.0/

\begin{abstract}
Background: Platelets play a key role in the development and progression of cardiovascular diseases. Also red cell distribution width (RDW\%) \& platelet indices are a good predictor of clinical outcomes. Purpose: Study the relationship between RDW\%, platelets count, mean platelet volume (MPVfl) and platelet distribution width (PDWfl) in children with congenital heart disease (CHD) or rheumatic heart diseases (RHD). Subjects and Methods: The study was carried on 151 children diagnosed as CHD or RHD selected from pediatric department of Al-Zahraa University Hospital and National Heart Institute. They were aged from 6 months to 12 years. Another 80 apparently healthy children were taken as controls. Complete blood count and echocardiography examination were evaluated for all participants. Results: The mean value of RDW\% was increased in CHD and RHD than controls, RDW\% higher in cyanotic CHD (CCHD) (either decompensated or compensated) than acyanotic CHD, and in decompensated RHD than compensated RHD with more than one valve affection. The mean platelets count were decreased in cyanotic than acyanotic CHD, platelets count were increased in decompensated than compensated RHD either with one valve or more than one valve affection. The mean values of MPV and PDW were increased in decompensated CHD, but it decreased in decompensated RHD. Conclusion: The RDW\%, MPV and PDW considered as simple markers in the follow up of patients with $\mathrm{CHD}$ or RHD for early detection of serious complication.
\end{abstract}

\section{Keywords}

Congenital, Disease, Heart, Platelet Indices, Red Cell, Distribution Width, Rheumatic Heart Diseases 


\section{Introduction}

Congenital heart disease (CHD) defined as a structural abnormality of the heart and (or) great vessels that is present at birth [1]. Congenital heart disease is one of the most frequently diagnosed congenital disorders affecting approximately $0.8 \%$ to $1.2 \%$ of live births worldwide [2].

Rheumatic heart disease is a chronic disease affecting the heart valves mainly resulting from recurrent severe attacks of acute rheumatic fever (ARF) [3], and considered as one of the most common leading causes of cardiovascular diseases mortality in developing countries in children, adolescent and young adults [4].

The RDW\% is a quantitative parameter representing red blood cells size and reflects heterogeneity in its volume [5]. RDW\% is used as an indicator of inflammation and potential marker of prognosis in cardiovascular events of various diseases [6].

Platelets have emerged as an important marker for various types of diseases. They are multifunctional blood particles and regarded to be very important clinical targets for many diseases pathophysiology. In addition to playing a central role in normal hemostasis and thrombosis, platelets can make important contributions to host inflammatory and immune responses to infection or injury. Under uncontrolled pathological conditions, they have profound roles in pathogenic processes underlying atherosclerosis, cardiovascular diseases and uncontrolled inflammation [7]. The MPV and PDW are potential biomarkers of cardiovascular diseases (CVD) [8].

\section{Aim of the Work}

To investigate the relationship between RDW\%, platelets count and its indices (MPV and PDW) in one side and chronic heart diseases either congenital or rheumatic on other side, also to investigate if there is a relationship between those parameters and severity of illness and decompensation in both RHD and CHD in order to promote early intervention and allow better out come in such patients.

\section{Patients and Methods}

This was a case controls study carried on 151 patients diagnosed as CHD or RHD aged from 6 months to 12 years old, selected from Al Zahraa University Hospital and National Heart Institute in the period from September 2018 to July 2019. Another 80 apparently healthy children age and sex matched with patients groups.

\subsection{Inclusion Criteria}

*Case groups:

- age: from 6 months to 12 years.

- sex: both sex were included.

The study population Included 151 cases subclassified into:

- Congenital heart disease group included 111case, 41case with CCHD and 70 
case with acyanotic CHD, both cyanotic and acyanotic CHD could be compensated or decompensated.

- Rheumatic heart disease group included 40 case, 17 case with one valve affection and 23 case with more than one valve lesion, both of them either decompensated or compensated.

*Controls:

Eighty apparently healthy children age and sex matched with patients groups.

\subsection{Exclusion Criteria}

- Any chronic disease other than rheumatic heart disease.

- Blood diseases.

- Other Chronic inflammation or infection.

- Obese children and adolescents were also excluded from this study.

\section{Methods}

All cases were subjected to complete history taking, general and cardiac examination, laboratory investigation in the form of complete blood count to study (RDW\%, MPV, PDW, platelets count), CRP, ESR and echocardiography examination using (Mindray M9 SC9101557, NYE 91102267, CC1-92000913) (GE Vivid S 5N, 0503788VS5N). MPV was measured within 30 minutes of sampling by Bechman Counter (USA) in a blood sample collected in citrate $(1: 4 \mathrm{v} / \mathrm{v})$ in order to avoid platelets swelling induced by EDTA.

\subsection{Statistical Analysis}

Recorded data were analyzed by statistical package for social sciences, version 20.0 (SPSS Inc., Chicago, Illinois, USA). Quantitative data were expressed as mean \pm standard deviation (SD) while qualitative data were expressed as frequency and percentage. Independent-samples t-test of significance was used for comparing between two means. A one-way analysis of variance (ANOVA) compared between more than two means. Hoc test used to detect differences in significant ANOVA test between multiple comparisons of different variables. While Chi-square $\left(\mathrm{X}^{2}\right)$ test was used in comparison between qualitative parameters. Receiver operating characteristic (ROC curve) analysis was used to predict severity in CHD and RHD, best cut-off value, sensitivity and specificity at this cut-off value. The confidence interval was set to $95 \%$ and $5 \%$ was the margin error. So, the P-value $<0.05$ was considered significant, $\mathrm{P}$-value $<0.001$ was highly significant and $\mathrm{P}$-value $>0.05$ was insignificant.

\subsection{Ethical Consideration}

The protocol of this study was approved by ethical committee of faculty of Medicine for Girls-Al-Azhar University.

An informed oral consent was obtained from all parents of patients before getting them involved in this study. 


\section{Results}

Regarding demographic data of cases and controls, there was no significant difference between them, and clinical data of patient showed that there was $91 \%$ of CHD patients on antifailure medication, $73.9 \%$ of them has pan systolic murmur (PSM) and $45.9 \%$ presented by shortness of breath, while $100 \%$ of RHD patients had history of repeated tonsillitis, $95 \%$ of them had history of long acting penicillin, 75\% has PSM and $47.5 \%$ presented by shortness of breath (Table 1(a), Table 1(b)).

In CHD subgroups, our study showed that RDW\%, MPV, PDW and CRP were higher, while the mean values of platelets count and ESR were lower in cases with cyanotic compared to acyanotic CHD (Table 2(a), Table 2(b)).

In RHD subgroups, also the mean values of RDW\%, platelets count, CRP and ESR were higher, while the mean values of MPV and PDW were lower in decompensated compared to compensated RHD either with one valve or more than one valve lesion (Table 3(a), Table 3(b)).

Table 1. (a) Demographic data of studied groups; (b) Clinical characteristics of studied cases.

(a)

\begin{tabular}{ccccc}
\hline Demographic Data & $\begin{array}{c}\text { Patients } \\
(\mathbf{n}=151)\end{array}$ & $\begin{array}{c}\text { Controls } \\
(\mathbf{n}=80)\end{array}$ & $\mathbf{t} / \mathbf{x} 2 \#$ & p-value \\
\hline $\begin{array}{c}\text { Age (years) } \\
\text { Mean } \pm \text { SD }\end{array}$ & $5.66 \pm 4.34$ & $6.58 \pm 4.30$ & 1.356 & 0.126 \\
Range & $0.5-12$ & $0.5-12$ & & \\
Sex & & & & \\
Female & $86(57.0 \%)$ & $54(67.5 \%)$ & $2.436 \#$ & \\
Male & $65(43.0 \%)$ & $26(32.5 \%)$ & & \\
\hline
\end{tabular}

(b)

\begin{tabular}{ccc}
\hline & Congenital & Rheumatic \\
\hline Frequency of the clinical findings & & \\
Shortness of breath & $51(45.9 \%)$ & $19(47.5 \%)$ \\
Cyanosis & $36(32.4 \%)$ & $0(0.0 \%)$ \\
History of recurrent cyanotic spells & $23(20.7 \%)$ & $0(0.0 \%)$ \\
Antifailure medication & $101(91.0 \%)$ & $23(57.5 \%)$ \\
Recurrent heart failure & $29(26.1 \%)$ & $2(5 \%)$ \\
History of repeated tonsillitis & $3(2.7 \%)$ & $40(100.0 \%)$ \\
History of long acting penicilline & $0(0.0 \%)$ & $38(95.0 \%)$ \\
Enlarged tender liver & $32(28.8 \%)$ & $8(20.0 \%)$ \\
Pan systolic murmur (PSM) & $82(73.9 \%)$ & $30(75.0 \%)$ \\
\hline
\end{tabular}


Table 2. (a) Comparison between congenital heart disease subgroups either decompensated or compensated as regard laboratory data readings; (b) Comparison between congenital heart disease subgroups either decompensated or compensated as regard laboratory data results using Post Hoc analysis: Tukey test.

(a)

\begin{tabular}{|c|c|c|c|c|c|c|c|}
\hline \multirow{2}{*}{ Laboratory data } & \multicolumn{2}{|c|}{ Decompensated CHD $(n=45)$} & \multicolumn{2}{|c|}{ Compensated CHD $(n=66)$} & \multirow{2}{*}{$\begin{array}{l}\text { Controls } \\
(n=80)\end{array}$} & \multirow[b]{2}{*}{$\mathbf{F}$} & \multirow[b]{2}{*}{ p-value } \\
\hline & $\begin{array}{c}\text { Cyanotic } \\
\text { CHD }(n=10)\end{array}$ & $\begin{array}{c}\text { Acyanotic } \\
\text { CHD }(n=35)\end{array}$ & $\begin{array}{c}\text { Cyanotic } \\
\text { CHD }(n=31)\end{array}$ & $\begin{array}{c}\text { Acyanotic } \\
\text { CHD }(n=35)\end{array}$ & & & \\
\hline \multicolumn{8}{|l|}{ RDW\% } \\
\hline Mean \pm SD & $17.01 \pm 4.57$ & $16.27 \pm 3.24$ & $14.45 \pm 2.72$ & $13.02 \pm 1.69$ & $11.31 \pm 1.12$ & \multirow{2}{*}{37.959} & \multirow{2}{*}{$<0.001^{*}$} \\
\hline Range & $13-25$ & $11.2-22.3$ & $11.1-21.4$ & $10.4-16.5$ & $8.5-13$ & & \\
\hline \multicolumn{8}{|c|}{ Platelets count $\times 10^{3} / \mu 1$} \\
\hline Mean \pm SD & $187.10 \pm 80.26$ & $310.77 \pm 145.98$ & $203.45 \pm 85.80$ & $351.86 \pm 104.72$ & $242.08 \pm 83.80$ & \multirow{2}{*}{13.424} & \multirow{2}{*}{$<0.001^{*}$} \\
\hline Range & 75 - 344 & $146-635$ & $68-342$ & $210-547$ & $4-362$ & & \\
\hline \multicolumn{8}{|l|}{ MPV fl } \\
\hline Mean \pm SD & $9.52 \pm 0.46$ & $8.97 \pm 0.99$ & $9.10 \pm 0.81$ & $7.72 \pm 0.85$ & $8.52 \pm 0.61$ & \multirow[b]{2}{*}{20.634} & \multirow[b]{2}{*}{$<0.001^{*}$} \\
\hline Range & $8.9-10$ & $6.9-10.9$ & $7.8-10.3$ & $5.1-9.1$ & $7.79-9.9$ & & \\
\hline \multicolumn{8}{|l|}{ PDW fl } \\
\hline Mean \pm SD & $13.81 \pm 2.33$ & $12.80 \pm 2.19$ & $14.26 \pm 2.25$ & $11.50 \pm 2.08$ & $12.06 \pm 1.13$ & \multirow{2}{*}{12.665} & \multirow[b]{2}{*}{$<0.001^{*}$} \\
\hline Range & $11-17$ & $10.3-20.5$ & $10.2-17$ & $8.3-16$ & $8.46-14.7$ & & \\
\hline \multicolumn{8}{|l|}{ CRP mg/l } \\
\hline Mean \pm SD & $43.21 \pm 16.79$ & $9.58 \pm 16.20$ & $5.51 \pm 6.56$ & $1.67 \pm 2.66$ & \multirow{2}{*}{$\begin{array}{l}\text { Standard } \\
\text { variation }\end{array}$} & \multirow{2}{*}{38.781} & \multirow{2}{*}{$<0.001^{* *}$} \\
\hline Range & $19-78$ & $0-58$ & $0-28$ & $0-10.19$ & & & \\
\hline \multicolumn{8}{|l|}{ ESR $\mathrm{mm} / \mathrm{h}$} \\
\hline Mean \pm SD & $14.67 \pm 8.91$ & $26.46 \pm 22.76$ & $6.44 \pm 7.37$ & $16.82 \pm 16.30$ & Standard & \multirow{2}{*}{8.169} & \multirow{2}{*}{$<0.001^{* *}$} \\
\hline Range & $1-24$ & $4-88$ & $0-37.7$ & $0-75$ & variation & & \\
\hline
\end{tabular}

${ }^{*}$ Denote significant, RDW\%: red cell distribution, MPV: mean platelet volume, PDW: platelet distribution width, fl: femtoliter, CRP: C-reactive protein, $\mathrm{mg} / \mathrm{l}$ : melligram per liter, ESR: erythrocyte sedimentation rate, $\mathrm{mm} / \mathrm{h}$ : millimeter per hour.

(b)

\begin{tabular}{|c|c|c|c|c|}
\hline & \multicolumn{4}{|c|}{ Post Hoc analysis: Tukey test } \\
\hline & Decompensated CHD & Compensate CHD & Cyanotic CHD & Acyanotic CHD \\
\hline & Cyanotic/Acyanotic & Cyanotic/Acyanotic & $\begin{array}{l}\text { Decompensated/ } \\
\text { Compensated }\end{array}$ & $\begin{array}{c}\text { Decompensated/ } \\
\text { Compensated }\end{array}$ \\
\hline RDW\% & 0.602 & $0.012^{*}$ & $0.007^{*}$ & $<0.001^{* *}$ \\
\hline Platelets $\times 10^{3} / \mu \mathrm{l}$ & $0.001^{*}$ & $<0.001^{* *}$ & 0.660 & 0.093 \\
\hline MPV fl & $0.046^{*}$ & $<0.001^{* *}$ & 0.130 & $<0.001^{* *}$ \\
\hline PDW fl & 0.121 & $<0.001^{* *}$ & 0.495 & $0.003^{*}$ \\
\hline CRP mg/l & $<0.001^{* *}$ & 0.159 & $<0.001^{* *}$ & $0.003^{*}$ \\
\hline ESR $\mathrm{mm} / \mathrm{h}$ & $0.048^{*}$ & $0.012^{*}$ & 0.172 & $0.016^{*}$ \\
\hline
\end{tabular}


Table 3. (a) Comparison between rheumatic heart disease subgroups either decompensated or compensated as regard laboratory data readings; (b) Comparison between rheumatic heart disease subgroups either decompensated or compensated as regard laboratory data results using Post Hoc analysis: Tukey test.

(a)

\begin{tabular}{|c|c|c|c|c|c|c|c|}
\hline \multirow[b]{2}{*}{$\mathrm{CBC}$} & \multicolumn{2}{|c|}{ Decompensated RHD $(n=17)$} & \multicolumn{2}{|c|}{ Compensated RHD (n=23) } & \multirow[b]{2}{*}{ Controls } & \multirow[b]{2}{*}{ F } & \multirow[b]{2}{*}{ p-value } \\
\hline & $\begin{array}{l}\text { RHD one valve } \\
\qquad(\mathrm{n}=8)\end{array}$ & $\begin{array}{l}\text { RHD more than } \\
\text { one valve }(n=9)\end{array}$ & $\begin{array}{l}\text { RHD one valve } \\
\qquad(\mathrm{n}=9)\end{array}$ & $\begin{array}{l}\text { RHD more than } \\
\text { one valve }(n=14)\end{array}$ & & & \\
\hline \multicolumn{8}{|l|}{ RDW\% } \\
\hline Mean \pm SD & $14.03 \pm 1.12$ & $14.76 \pm 1.54$ & $13.03 \pm 1.95$ & $13.57 \pm 0.50$ & $11.31 \pm 1.12$ & \multirow{2}{*}{32.519} & \multirow{2}{*}{$<0.001^{\star *}$} \\
\hline Range & $12.6-15.1$ & $12.3-17.4$ & $10.4-17$ & $12.6-14.1$ & $8.5-13$ & & \\
\hline \multicolumn{8}{|c|}{ Platelets count $\times 10^{3} / \mu 1$} \\
\hline Mean \pm SD & $377.38 \pm 32.73$ & $396.33 \pm 17.56$ & $310.33 \pm 52.13$ & $335.36 \pm 32.03$ & $242.08 \pm 83.80$ & \multirow[b]{2}{*}{17.431} & \multirow[b]{2}{*}{$<0.001^{\star *}$} \\
\hline Range & $340-440$ & $380-430$ & $227-411$ & $278-376$ & $4-362$ & & \\
\hline \multicolumn{8}{|l|}{ MPV fl } \\
\hline Mean \pm SD & $8.15 \pm 0.20$ & $7.72 \pm 0.66$ & $8.68 \pm 0.44$ & $8.71 \pm 0.48$ & $8.52 \pm 0.61$ & \multirow[b]{2}{*}{5.608} & \multirow[b]{2}{*}{$<0.001^{* *}$} \\
\hline Range & $7.7-8.3$ & $6.8-9$ & $7.8-9.2$ & $8.3-9.8$ & $7.79-9.9$ & & \\
\hline \multicolumn{8}{|l|}{ PDW fl } \\
\hline Mean \pm SD & $11.05 \pm 1.21$ & $10.63 \pm 1.67$ & $12.42 \pm 1.80$ & $13.02 \pm 1.04$ & $12.06 \pm 1.13$ & \multirow[b]{2}{*}{6.723} & \multirow[b]{2}{*}{$<0.001^{\star *}$} \\
\hline Range & $10-13.4$ & $7.1-13.5$ & $8.8-14.8$ & $11.2-14.7$ & $8.46-14.7$ & & \\
\hline \multicolumn{8}{|l|}{ CRP mg/l } \\
\hline Mean \pm SD & $29.73 \pm 22.57$ & $34.29 \pm 34.90$ & $4.22 \pm 4.31$ & $5.96 \pm 3.51$ & \multirow{2}{*}{$\begin{array}{l}\text { Standard } \\
\text { variation }\end{array}$} & \multirow{2}{*}{6.307} & \multirow{2}{*}{$0.002^{*}$} \\
\hline Range & $8.8-79$ & $10-105$ & $0-11$ & $1.5-9.2$ & & & \\
\hline \multicolumn{8}{|l|}{$\mathrm{ESR} \mathrm{mm} / \mathrm{h}$} \\
\hline Mean \pm SD & $45.38 \pm 9.40$ & $67.89 \pm 39.24$ & $8.19 \pm 5.05$ & $10.13 \pm 3.72$ & \multirow{2}{*}{$\begin{array}{l}\text { Standard } \\
\text { variation }\end{array}$} & \multirow{2}{*}{22.174} & \multirow{2}{*}{$<0.001^{* *}$} \\
\hline Range & $33-58$ & $13-125$ & $4.1-17$ & $6-17.4$ & & & \\
\hline
\end{tabular}

(b)

\begin{tabular}{|c|c|c|c|c|}
\hline & \multicolumn{4}{|c|}{ Post Hoc analysis: Tukey test } \\
\hline & Decompensated RHD & Compensated RHD & RHD one valve & RHD two valve \\
\hline & Two valve/One valve & Two valve/One valve & $\begin{array}{l}\text { Decompensated/ } \\
\text { Compensated }\end{array}$ & $\begin{array}{l}\text { Decompensated/ } \\
\text { Compensated }\end{array}$ \\
\hline RDW\% & 0.205 & 0.288 & 0.086 & $0.021^{*}$ \\
\hline Platelets $10^{3} / \mu \mathrm{l}$ & 0.590 & 0.419 & $0.059^{*}$ & $0.051^{*}$ \\
\hline MPV fl & 0.126 & 0.881 & 0.060 & $<0.001^{* *}$ \\
\hline PDW fl & 0.486 & 0.255 & $0.023^{*}$ & $0.001^{* *}$ \\
\hline CRP mg/l & 0.632 & 0.836 & $0.011^{*}$ & $0.002^{*}$ \\
\hline ESR $\mathrm{mm} / \mathrm{h}$ & $0.021^{*}$ & 0.815 & $<0.001^{\star *}$ & $<0.001^{* *}$ \\
\hline
\end{tabular}


In $\mathrm{CHD}$ cases the RDW\%, MPV and PDW levels were significantly higher compared to both RHD and controls. The mean platelets count increased in RHD in comparison to either CHD or controls. Increase CRP and ESR in RHD than CHD (Table 4(a)-(c)).

The mean values of RDW\%, MPV and PDW increase in decompensated CHD than decompensated RHD, while increase platelets count, CRP and ESR in decompensated RHD compared to decompensated CHD (Table 5(a), Table 5(b)).

Our results also, showed that there was a significant positive correlation between RDW\%, MPV, CRP and ESR with left ventricular end diastolic dimension (LVEDD) and left ventricular end systolic dimension (LVESD), while negative correlation with ejection fraction (EF\%) in CHD cases (Table 6).

Also our study showed that, positive correlation between RDW\% with LVESD and pulmonary artery pressure (PAP), were negative correlation with EF\%. The MPV, PDW correlated negatively with LVEDD, LVESD and PAP, while correlated positively with EF\%. Platelets count correlated positively with LVEDD and LVESD, and negatively with EF\%. No significant correlation between Platelets count with PAP in RHD cases (Table 7).

The results of the ROC curve analysis revealed that RDW\% cut off point was > 13.8\% (Table 8). Also the best cut-off point regarding platelets count was less than $295 \times 10^{3} / \mu \mathrm{l}$, MPVfl was more than $8.7 \mathrm{fl}$ and PDWfl was found more than $13.5 \mathrm{fl}$. So it could be considered as useful markers in the follow up assessment of CHD and RHD patients for early detection of complication (Table 9) (Figure 1, Figure 2).

\section{Discussion}

The present study showed that increased RDW\% value in all cases with CHD than controls. Cases with CHD in the presence of heart failure (HF), showed significant increase of RDW\% in all cases with decompensated CHD in comparison to cases with compensated CHD, also significant increase of RDW\% in decompensated cases either with (CCHD or acyanotic CHD).

These results come in line with a previous study which reported that preoperative elevated RDW\% is a novel and strong predictor of adverse outcomes for children undergoing cardiac surgery for CHD [9], which was similar to the results of Polat et al. [10].

Also agreed with our results, Mawlana et al., evaluated the relation between RDW\% and left ventricular function in children with HF, and found that RDW\% higher in those patients [11].

This observation can be explained by that erythrocytes are more vulnerable to the effects of oxidative damage. Oxidative stress decreases production of erythropoietin and aggravates destructive process of erythrocytes leading to ineffective production of red blood cells. Erythrocyte damage decreases in the life cycle of available erythrocytes, and entrance of the immature erythrocytes into the general circulation increases level of RDW\% [12]. 
Table 4. (a) Comparison between all studied groups as regard CBC parameters readings; (b) Comparison of CBC parameters results between all studied groups using Post Hoc analysis: Tukey test; (c) Comparison between all congenital and rheumatic heart disease groups as regard inflammatory markers readings and results.

(a)

\begin{tabular}{|c|c|c|c|c|c|}
\hline $\mathrm{CBC}$ & Congenital $(n=111)$ & Rheumatic $(n=40)$ & Controls $(\mathrm{n}=80)$ & $\mathbf{F}$ & p-value \\
\hline \multicolumn{6}{|l|}{ RDW\% } \\
\hline Mean \pm SD & $14.78 \pm 3.15$ & $13.81 \pm 1.40$ & $11.31 \pm 1.12$ & & \\
\hline Range & $10.4-25$ & $10.4-17.4$ & $8.5-13$ & 51.074 & $<0.001^{* *}$ \\
\hline \multicolumn{6}{|c|}{ Platelets count $\times 10^{3} / \mu 1$} \\
\hline Mean \pm SD & $282.61 \pm 129.94$ & $351.85 \pm 47.34$ & $242.08 \pm 83.80$ & & \\
\hline Range & $68-635$ & $227-440$ & $4-362$ & 14.697 & $<0.001^{* *}$ \\
\hline \multicolumn{6}{|l|}{ MPV fl } \\
\hline Mean \pm SD & $8.66 \pm 1.07$ & $8.37 \pm 0.62$ & $8.52 \pm 0.61$ & & \\
\hline Range & $5.1-10.9$ & $6.8-9.8$ & $7.79-9.9$ & 1.786 & 0.170 \\
\hline \multicolumn{6}{|l|}{ PDW fl } \\
\hline Mean \pm SD & $12.89 \pm 2.42$ & $11.96 \pm 1.70$ & $12.06 \pm 1.13$ & & \\
\hline & & & & 5.719 & $0.004^{*}$ \\
\hline Range & $8.3-20.5$ & $7.1-14.8$ & $8.46-14.7$ & & \\
\hline
\end{tabular}

(b)

Post Hoc analysis: Tukey test

Congenital/controls

Rheumatic/controls

Congenital/rheumatic

\begin{tabular}{cccc}
\hline RDW\% & $0.027^{*}$ & $<0.001^{* *}$ & $<0.001^{* *}$ \\
Platelets $\times 10^{3} / \mu 1$ & $<0.001^{* *}$ & $0.009^{*}$ & $<0.001^{* *}$ \\
MPV fl & $0.007^{*}$ & 0.282 & 0.358 \\
PDW fl & $0.010^{*}$ & $\mathbf{0 . 0 0 4 ^ { * }}$ & 0.779 \\
\hline
\end{tabular}

(c)

\begin{tabular}{|c|c|c|c|c|}
\hline Inflammatory markers & Congenital $(n=111)$ & Rheumatic $(n=40)$ & t-test & p-value \\
\hline \multicolumn{5}{|l|}{ CRP mg/l } \\
\hline \multirow[t]{2}{*}{ Mean \pm SD } & $8.97 \pm 15.65$ & $16.70 \pm 23.08$ & & \\
\hline & & & -2.335 & $0.021^{*}$ \\
\hline Range & $0-78$ & $0-105$ & & \\
\hline \multicolumn{5}{|l|}{$\mathrm{ESR} \mathrm{mm} / \mathrm{h}$} \\
\hline \multirow[t]{2}{*}{ Mean \pm SD } & $16.76 \pm 18.00$ & $29.74 \pm 31.19$ & & \\
\hline & & & -3.166 & $0.002^{*}$ \\
\hline Range & $0-88$ & $4.1-125$ & & \\
\hline
\end{tabular}


Table 5. (a) Comparison between all congenital and rheumatic heart disease either decompensated or compensated as regard laboratory data readings; (b) Comparison between congenital and rheumatic heart disease groups either compensated or decompensated as regard laboratory data results using Post Hoc analysis: Tukey test.

(a)

\begin{tabular}{|c|c|c|c|c|c|c|}
\hline & \multicolumn{2}{|c|}{ Decompensated heart disease } & \multicolumn{2}{|c|}{ Compensated heart disease } & \multirow{2}{*}{ F } & \multirow{2}{*}{ p-value } \\
\hline & $\mathrm{CHD}(\mathrm{n}=45)$ & RHD ( $n=17)$ & $\mathrm{CHD}(\mathrm{n}=66)$ & RHD (n = 23) & & \\
\hline \multicolumn{7}{|l|}{ RDW\% } \\
\hline Mean \pm SD & $616.37 \pm 3.52$ & $14.41 \pm 1.37$ & $13.69 \pm 2.33$ & $13.36 \pm 1.27$ & \multirow[b]{2}{*}{40.919} & \multirow[b]{2}{*}{$<0.001^{* *}$} \\
\hline Range & $11.2-25$ & $12.3-17.4$ & $10.4-21.4$ & $10.4-17$ & & \\
\hline \multicolumn{7}{|c|}{ Platelets count $\times 10^{3} / \mu 1$} \\
\hline Mean \pm SD & $283.29 \pm 143.14$ & $387.41 \pm 26.80$ & $282.15 \pm 121.26$ & $325.57 \pm 41.84$ & \multirow[b]{2}{*}{8.254} & \multirow[b]{2}{*}{$<0.001^{* *}$} \\
\hline Range & $75-635$ & $340-440$ & $68-547$ & $227-411$ & & \\
\hline \multicolumn{7}{|l|}{ MPV fl } \\
\hline Mean \pm SD & $9.09 \pm 0.93$ & $7.92 \pm 0.53$ & $8.37 \pm 1.08$ & $8.70 \pm 0.46$ & \multirow[b]{2}{*}{8.426} & \multirow[b]{2}{*}{$<0.001^{\star *}$} \\
\hline Range & $6.9-10.9$ & $6.8-9$ & $5.1-10.3$ & $7.8-9.8$ & & \\
\hline \multicolumn{7}{|l|}{ PDW fl } \\
\hline Mean \pm SD & $13.02 \pm 2.24$ & $10.83 \pm 1.44$ & $12.80 \pm 2.56$ & $12.79 \pm 1.38$ & \multirow[b]{2}{*}{5.641} & \multirow[b]{2}{*}{$<0.001^{*}$} \\
\hline Range & $10.3-20.5$ & $7.1-13.5$ & $8.3-17$ & $8.8-14.8$ & & \\
\hline \multicolumn{7}{|l|}{ CRP mg/l } \\
\hline Mean \pm SD & $17.22 \pm 21.53$ & $32.14 \pm 28.94$ & $3.47 \pm 5.23$ & $5.28 \pm 3.84$ & \multirow[b]{2}{*}{18.942} & \multirow[b]{2}{*}{$<0.001^{* *}$} \\
\hline Range & $0-78$ & $8.8-105$ & $0-28$ & $0-11$ & & \\
\hline \multicolumn{7}{|l|}{$\mathrm{ESR} \mathrm{mm} / \mathrm{h}$} \\
\hline Mean \pm SD & $23.84 \pm 21.00$ & $57.29 \pm 30.71$ & $11.94 \pm 13.83$ & $9.37 \pm 4.29$ & \multirow[b]{2}{*}{32.284} & \multirow[b]{2}{*}{$<0.001^{* *}$} \\
\hline Range & $1-88$ & $13-125$ & $0-75$ & $4.1-17.4$ & & \\
\hline
\end{tabular}

(b)

\begin{tabular}{|c|c|c|c|c|}
\hline & \multicolumn{4}{|c|}{ Post Hoc analysis: Tukey test } \\
\hline & $\begin{array}{l}\text { Decompensated } \\
\text { heart disease }\end{array}$ & $\begin{array}{l}\text { Compensated } \\
\text { heart disease }\end{array}$ & CHD & RHD \\
\hline & CHD/RHD & CHD/RHD & $\begin{array}{l}\text { Decompensated/ } \\
\text { Compensated }\end{array}$ & $\begin{array}{c}\text { Decompensated/ } \\
\text { Compensated }\end{array}$ \\
\hline RDW\% & $0.002^{*}$ & 0.530 & $<0.001^{* *}$ & 0.131 \\
\hline Platelets $\times 10^{3} / \mu \mathrm{l}$ & $0.001^{*}$ & 0.087 & 0.955 & 0.065 \\
\hline MPV fl & $<0.001^{* *}$ & 0.095 & $<0.001^{* *}$ & $0.003^{*}$ \\
\hline PDW fl & $<0.001^{* *}$ & 0.985 & 0.539 & $0.002^{*}$ \\
\hline CRP mg/l & $0.001^{*}$ & 0.633 & $<0.001^{* *}$ & $<0.001^{* *}$ \\
\hline ESR $\mathrm{mm} / \mathrm{h}$ & $<0.001^{* *}$ & 0.555 & $0.001^{*}$ & $<0.001^{* *}$ \\
\hline
\end{tabular}


Table 6. Correlation study between laboratory data with age and clinical findings of all cases with congenital heart disease.

\begin{tabular}{|c|c|c|c|c|c|c|c|c|c|c|}
\hline \multicolumn{2}{|c|}{ Congenital } & \multirow{2}{*}{$\begin{array}{c}\text { RBC } \\
\times 10^{6} / \mu \mathrm{l}\end{array}$} & \multirow{2}{*}{$\begin{array}{l}\mathrm{Hb} \text { g/dl } \\
-0.102\end{array}$} & \multirow{2}{*}{$\begin{array}{l}\text { HTC\% } \\
-0.125\end{array}$} & \multirow{2}{*}{$\begin{array}{c}\text { RDW\% } \\
0.093\end{array}$} & \multirow{2}{*}{$\begin{array}{c}\begin{array}{c}\text { Platelets } \\
\times 10^{3} / \mu 1\end{array} \\
0.022\end{array}$} & \multirow{2}{*}{$\begin{array}{l}\text { MPV fl } \\
-0.068\end{array}$} & \multirow{2}{*}{$\begin{array}{c}\text { PDW fl } \\
0.027\end{array}$} & \multirow{2}{*}{$\begin{array}{c}\text { CRP mg/l } \\
0.078\end{array}$} & \multirow{2}{*}{$\begin{array}{c}\text { ESR mm/h } \\
-0.016\end{array}$} \\
\hline & $r$-value & & & & & & & & & \\
\hline & $\mathrm{p}$-value & 0.692 & 0.285 & 0.191 & 0.333 & 0.823 & 0.481 & 0.777 & 0.421 & 0.865 \\
\hline \multirow{2}{*}{$\mathrm{RR} \mathrm{C} / \mathrm{M}$} & r-value & 0.017 & 0.063 & 0.113 & 0.150 & -0.278 & 0.122 & 0.025 & 0.203 & -0.068 \\
\hline & $\mathrm{p}$-value & 0.863 & 0.509 & 0.239 & 0.117 & $0.003^{*}$ & 0.201 & 0.796 & $0.033^{*}$ & 0.481 \\
\hline \multirow{2}{*}{$\mathrm{HR} \mathrm{b} / \mathrm{m}$} & $\mathrm{r}$-value & 0.007 & -0.194 & -0.106 & 0.205 & -0.129 & 0.004 & -0.001 & 0.280 & -0.092 \\
\hline & $\mathrm{p}$-value & 0.940 & $0.041^{*}$ & 0.269 & $0.031^{*}$ & 0.176 & 0.967 & 0.990 & $0.003^{*}$ & 0.335 \\
\hline \multirow{2}{*}{ LVEDD } & $\mathrm{r}$-value & -0.300 & -0.356 & -0.335 & 0.384 & 0.043 & 0.285 & 0.059 & 0.308 & 0.298 \\
\hline & $\mathrm{p}$-value & $0.001^{*}$ & $<0.001^{* *}$ & $<0.001^{* *}$ & $<0.001^{* *}$ & 0.658 & $0.003^{*}$ & 0.541 & $0.001^{*}$ & $0.002^{*}$ \\
\hline \multirow{2}{*}{ LVESD } & r-value & -0.341 & -0.422 & -0.394 & 0.416 & -0.004 & 0.315 & 0.046 & 0.286 & 0.282 \\
\hline & $\mathrm{p}$-value & $<0.001^{* *}$ & $<0.001^{* *}$ & $<0.001^{* *}$ & $<0.001^{* *}$ & 0.969 & $0.001^{*}$ & 0.628 & $0.002^{*}$ & $0.003^{*}$ \\
\hline \multirow{2}{*}{ PAP } & r-value & 0.286 & 0.192 & 0.270 & 0.021 & -0.079 & 0.118 & 0.222 & -0.006 & -0.134 \\
\hline & p-value & $0.002^{*}$ & $0.043^{*}$ & $0.004^{*}$ & 0.826 & 0.412 & 0.219 & $0.019^{*}$ & 0.952 & 0.160 \\
\hline \multirow{2}{*}{$\mathrm{EF} \%$} & r-value & 0.373 & 0.439 & 0.389 & -0.372 & -0.060 & -0.237 & 0.036 & -0.320 & -0.325 \\
\hline & $\mathrm{p}$-value & $<0.001^{\star *}$ & $<0.001^{* *}$ & $<0.001^{* *}$ & $<0.001^{* *}$ & 0.530 & $0.012^{*}$ & 0.706 & $0.001^{*}$ & $0.001^{*}$ \\
\hline
\end{tabular}

RR: respiratory rate, HR: heart rate, LVEDD: Left Ventericular End Diastolic diameter, LVESD: Left Ventericular End Systolic diameter, PAP: Pulmonary Artery Pressure, EF\%: Ejection Fraction\%.

Table 7. Correlation study between laboratory data with age and clinical findings of all cases with rheumatic heart disease.

\begin{tabular}{|c|c|c|c|c|c|c|c|c|c|c|}
\hline \multicolumn{2}{|c|}{ Rheumatic } & \multirow{2}{*}{$\begin{array}{c}\begin{array}{c}\text { RBC } \\
\times 10^{6} / \mu 1\end{array} \\
-0.093\end{array}$} & \multirow{2}{*}{$\begin{array}{l}\mathrm{Hb} \text { g/dl } \\
-0.158\end{array}$} & \multirow{2}{*}{$\begin{array}{r}\text { HTC\% } \\
-0.084\end{array}$} & \multirow{2}{*}{$\begin{array}{c}\text { RDW\% } \\
0.128\end{array}$} & \multirow{2}{*}{$\begin{array}{c}\begin{array}{c}\text { Platelets } \\
\times 10^{3} / \mu 1\end{array} \\
0.287\end{array}$} & \multirow{2}{*}{$\begin{array}{l}\text { MPV fl } \\
-0.112\end{array}$} & \multirow{2}{*}{$\begin{array}{c}\text { PDW fl } \\
0.049\end{array}$} & \multirow{2}{*}{$\begin{array}{c}\text { CRP mg/l } \\
0.045\end{array}$} & \multirow{2}{*}{$\begin{array}{c}\mathrm{ESR} \mathrm{mm} / \mathrm{h} \\
0.082\end{array}$} \\
\hline & r-value & & & & & & & & & \\
\hline & $\mathrm{p}$-value & 0.568 & 0.331 & 0.607 & 0.433 & 0.073 & 0.493 & 0.764 & 0.781 & 0.616 \\
\hline \multirow{2}{*}{ RR C/M } & r-value & -0.643 & -0.347 & -0.434 & 0.347 & 0.607 & -0.609 & -0.505 & 0.508 & 0.679 \\
\hline & $\mathrm{p}$-value & $<0.001^{* *}$ & $0.028^{*}$ & $0.005^{*}$ & $0.028^{*}$ & $<0.001^{* *}$ & $<0.001^{* *}$ & $0.001^{*}$ & $0.001^{*}$ & $<0.001^{* *}$ \\
\hline \multirow{2}{*}{$\mathrm{HR} \mathrm{b} / \mathrm{m}$} & r-value & -0.492 & -0.222 & -0.286 & 0.306 & 0.553 & -0.594 & -0.452 & 0.415 & 0.636 \\
\hline & $\mathrm{p}$-value & $0.001^{*}$ & 0.168 & 0.073 & $0.055^{*}$ & $<0.001^{\star *}$ & $<0.001^{* *}$ & $0.003^{*}$ & $0.008^{*}$ & $<0.001^{* *}$ \\
\hline \multirow{2}{*}{ LVEDD } & r-value & -0.411 & -0.155 & -0.211 & 0.278 & 0.452 & -0.315 & -0.337 & 0.149 & 0.461 \\
\hline & $\mathrm{p}$-value & $0.008^{*}$ & 0.340 & 0.191 & 0.082 & $0.003^{*}$ & $0.048^{*}$ & $0.033^{*}$ & 0.360 & $0.003^{*}$ \\
\hline \multirow{2}{*}{ LVESD } & r-value & -0.563 & -0.416 & -0.394 & 0.389 & 0.557 & -0.459 & -0.396 & 0.476 & 0.614 \\
\hline & $\mathrm{p}$-value & $<0.001^{* *}$ & $0.008^{*}$ & $0.012^{*}$ & $0.013^{*}$ & $<0.001^{\star *}$ & $0.003^{*}$ & $0.011^{*}$ & $0.002^{*}$ & $<0.001^{* *}$ \\
\hline \multirow{2}{*}{ PAP } & r-value & -0.283 & -0.224 & -0.162 & 0.457 & 0.264 & -0.419 & -0.369 & 0.083 & 0.469 \\
\hline & $\mathrm{p}$-value & 0.077 & 0.164 & 0.319 & $0.003^{*}$ & 0.100 & $0.007^{*}$ & $0.019^{*}$ & 0.612 & $0.002^{*}$ \\
\hline \multirow{2}{*}{ EF\% } & r-value & 0.640 & 0.335 & 0.391 & -0.312 & -0.643 & 0.564 & 0.482 & -0.444 & -0.677 \\
\hline & $\mathrm{p}$-value & $<0.001^{* *}$ & $0.035^{*}$ & $0.013^{*}$ & $0.050^{*}$ & $<0.001^{\star *}$ & $<0.001^{* *}$ & $0.002^{*}$ & $0.004^{*}$ & $<0.001^{* *}$ \\
\hline
\end{tabular}


Table 8. Cut off point for RBC parameters and indices as predictor of severity in chronic heart disease either congenital or rheumatic heart disease.

\begin{tabular}{ccccccc}
\hline $\begin{array}{c}\text { RBC count } \\
\text { and RBC } \\
\text { indices }\end{array}$ & Cut-off & Sensitivity & Specificity & $\begin{array}{c}\text { Positive } \\
\text { predictive } \\
\text { value (PPV) }\end{array}$ & $\begin{array}{c}\text { Negative } \\
\text { predictive } \\
\text { value (NPV) }\end{array}$ & $\begin{array}{c}\text { Area under } \\
\text { the curve } \\
(\text { AUC) }\end{array}$ \\
\hline HTC\% & $>34$ & $43.2 \%$ & $70 \%$ & $80 \%$ & $30.8 \%$ & 0.542 \\
$\mathrm{MCV} \mathrm{fl}$ & $<74$ & $55.8 \%$ & $42.5 \%$ & $73 \%$ & $45 \%$ & 0.541 \\
$\mathrm{RDW} \%$ & $>13.8$ & $51.4 \%$ & $57.5 \%$ & $77 \%$ & $48.2 \%$ & 0.542 \\
$\mathrm{RBC} \times 10^{6} / \mu \mathrm{l}$ & $>4.48$ & $58.6 \%$ & $50 \%$ & $76.5 \%$ & $40.3 \%$ & 0.591 \\
$\mathrm{Hb}$ g/dl & $>11.4$ & $48.2 \%$ & $52.5 \%$ & $74.1 \%$ & $36.9 \%$ & 0.501 \\
\hline
\end{tabular}

Table 9. Cut off point for platelets count and platelet indices as predictor for severity in chronic heart disease cases either congenital or rheumatic heart disease.

\begin{tabular}{ccccccc}
\hline $\begin{array}{c}\text { Platelets count and } \\
\text { platelet indices }\end{array}$ & Cut-off & Sen. & Spe. & PPV & NPV & AUC \\
\hline Platelets $\times 10^{3} / \mu l$ & $<295$ & $61.3 \%$ & $92.5 \%$ & $95.8 \%$ & $46.3 \%$ & 0.742 \\
MPV fl & $>8.7$ & $47.7 \%$ & $82.5 \%$ & $88.3 \%$ & $36.3 \%$ & 0.597 \\
PDW fl & $>13.5$ & $36.0 \%$ & $87.5 \%$ & $88.9 \%$ & $33 \%$ & 0.589 \\
\hline
\end{tabular}

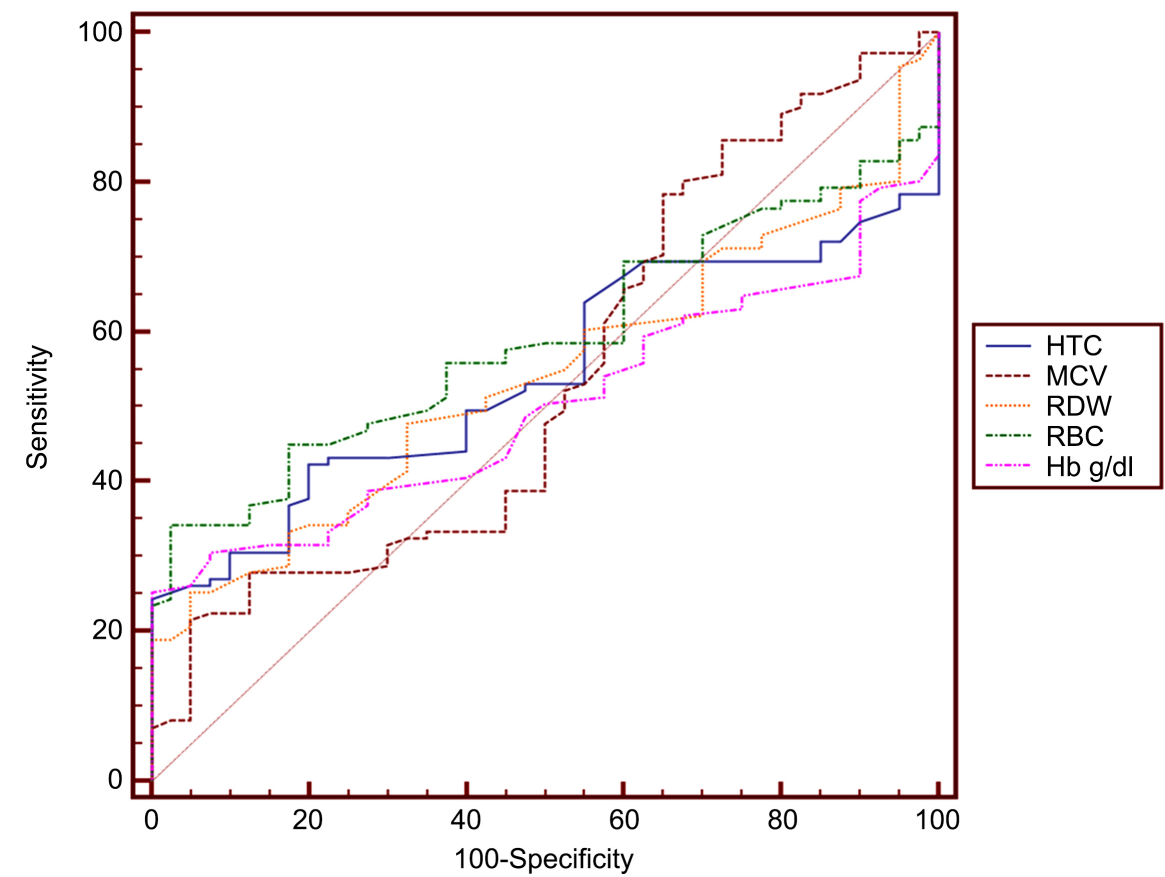

Figure 1. Receiver operating characteristic curve (ROC) of RBCs and RBC indices as predictor of severity in congenital and rheumatic heart disease. 


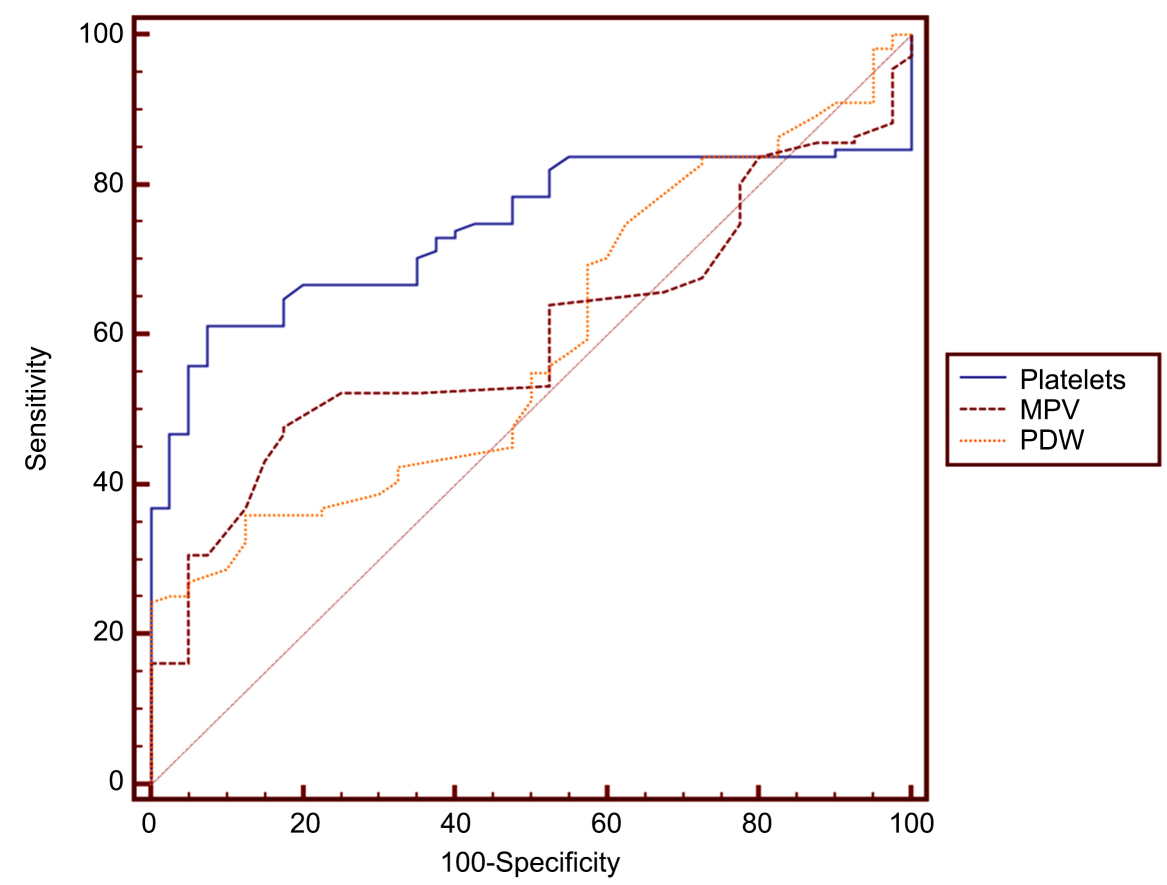

Figure 2. Receiver operating characteristic curve (ROC) of platelets count and platelet indices as predictor of severity in congenital and rheumatic heart disease.

In the present study children with compensated CCHD showed significantly higher value of RDW\% in comparison to children with acyanotic heart disease.

This result agreed with Animasahun et al. who found that, RDW\% was significantly higher in children with CCHD compared to controls [13]. Increased RDW\% is an indicator of the variation in erythrocyte volume which is expected to be increased when there is an exaggerated hematopoietic response as found in children with CCHD [14].

In the present study, children with CHD showed significant positive correlation between RDW\% with LVEDD and LVESD. While EF\% showed significant negative correlation with RDW\%, which means that left ventricular failure in children with CHD (as reflected in Echo by increased dimensions LVEDD, LVESD and decreased EF\%) causes significant increase of RDW\%.

Mawlana et al. stated that, RDW\% significantly correlated with Echo parameters for evaluation of left ventricular function in children with heart failure. So RDW\% considered as a simple, available test, can be used as a marker for the left ventricular failure in children with CHD until an echo assessment for the patients is done [11].

Also, Yudha et al., found a relationship between RDW\% and the parameters of left ventricular function in patients with acyanotic CHD, also found a relationship between RDW\% with EF\% in patients with atrial septal defect [15].

Yang et al., mentioned that a higher RDW\% was independently correlated with a lower left ventricular ejection fraction (LVEF\%) among hypertrophic cardiomyopathy patients with HF [16], which was similar to the results of Al-Najjar et al. [17]. 
This results could be explained by activation of the renin-angiotensin system in HF which associated with increased erythropoiesis lead to increase RDW\% [18].

As regard mean platelets count in studied cases with CHD, our study showed increases the mean value of platelets count in all cases with CHD in comparison to controls, but in cases with CHD complicated with HF the mean platelets count decreases especially in those patients with cyanotic $(187.10 \pm 80.26)$ in comparison to acyanotic CHD (310.77 \pm 145.98$)$.

Even in compensated CHD cases presence of cyanosis causes significant decreases in mean platelets count $(203.45 \pm 85.80)$ in comparison to CHD cases without cyanosis $(351.86 \pm 104.72)$.

Animasahun et al. found that, lower values of platelets count noticed in CCHD patients compared to the controls [13]. The pathogenesis of thrombocytopenia in cyanotic disease conditions are decreases platelets and megakaryocyte production, increase platelets activation and destruction. These mechanisms occur largely from reduced platelet production in the lung beds from less shunting of blood through the pulmonary veins in cyanotic heart diseases and delivery of megakaryocytes to the systemic arterial circulation through the shunt [19].

The MPV and PDW are markers of platelets activation. In the present study, there was significant increase in the mean values of MPV and PDW in all cases with CHD compared to controls.

The present study results showed that there was an effect of HF within CHD cases, the mean value of MPV increases in all cases with decompensated CHD compared to cases with compensated CHD.

In acyanotic CHD with HF, significant increase in MPV and PDW in cases with decompensated acyanotic CHD than cases with compensated acyanotic CHD.

Also our results showed that the presence of HF in congenital cyanotic and acyanotic heart disease, causes significant increase of MPV in cases with cyanotic compared to cases with acyanotic CHD.

Even in the absence of HF we found that, the mean values of MPV and PDW increased in cases with CCHD compared to cases with acyanotic CHD.

Mese et al. mentioned that, MPV and PDW were increased in children with $\mathrm{CHD}$ associated pulmonary arterial hypertension $(\mathrm{PAH})$ in comparison to cases without PAH and controls, they might give clue about disease severity [20].

Also Kaya et al. found that, MPV level were higher in the atrial septal defect (ASD) group than the control group [21].

Sato et al. found that, increase MPV and PDW in CHD patients with HF, and decrease MPV and PDW after treatment of HF [22].

Endothelial dysfunction in the setting of PAH may lead to increase procoagulant activity, inappropriate fibrinolysis and platelet activation [23]. Endothelial dysfunction may also result in an imbalance of vasoactive mediators. Proaggregatory thromboxane A2 level was increased, whereas nitric oxide and prostacyc- 
lin (inhibit platelet aggregation) levels are decreased in patients with PAH [24].

The present study showed positive correlation between MPV with both LVEDD and LVESD, and negative correlation with EF\%. While, there was positive correlation between PDW with PAP in CHD cases.

Sato et al. stated that, platelet volume indices correlated to severity of HF and have prognostic value for both cardiac and thrombotic events in patients with CHD [22].

Açikgöz et al. found that, Patients with idiopathic or ischemic cardiomyopathy have higher MPV values indicating tendency to platelet aggregation regardless of the etiology, when compared to controls and an enlarged dysfunctional left ventricle may also be associated with higher MPV values [25].

In addition, Kaya et al. mentioned that, MPV correlated positively with systolic PAP and right ventricular diameter in ASD patients [21].

Our study showed that significant increase of CRP and ESR in all cases with decompensated CHD compared to cases with compensated CHD. Also an significant increase of CRP and ESR in cases with decompensated acyanotic CHD compared to cases with compensated acyanotic $\mathrm{CHD}$ was detected.

As well as, increase CRP in cases with decompensated CCHD compared to cases with both compensated CCHD and cases with decompensated acyanotic CHD.

The mean values of ESR lower in cases with CCHD either (decompensated or compensated) compared to cases with acyanotic CHD.

In cases with $\mathrm{CHD}$, the current study results showed that positive correlation between CRP and ESR with LVEDD and LVESD. While, there was negative correlation between CRP with $\mathrm{EF} \%$.

Nassef et al. detected significantly elevated levels of CRP in CHD, with percentage increase in cyanotic than acyanotic patient as compared to the normal one [26]. The significant elevation in CRP is in a good agreement with Kantor and Rusconi, they found that CRP is associated with symptom severity, and able to discriminate between clinical severity groups [27].

Rheumatic carditis is an autoimmune inflammatory disorder that develops due to the body's abnormal and exaggerated immune response against beta hemolytic streptococci. The disease occurs through a cytokine mediated inflammatory reaction in which both cellular and humoral immune mechanisms play a role in susceptible individuals [28].

As regard cases with RHD, our study showed that significantly higher RDW\% value in all cases with RHD than controls.

Also our study showed that the effect of HF within cases with RHD, significant increase RDW\% in cases with decompensated RHD than cases with compensated RHD with more than one valve affection.

Karpuz et al. stated that, increase of RDW\% in patients with RHD compared to controls, suggests that RDW\% is a marker of the underlying chronic inflammation, which increases the risk of cardiovascular disease [29]. 
Also Kucuk et al. mentioned that RDW\% was significantly higher in patients with acute rheumatic carditis (ARC) compared with healthy controls, both at the time of diagnosis and after medical therapy. Moreover, RDW\% levels increased with the severity of the mitral regurgitation. They also discovered that RDW\% is higher in rheumatic carditis patients with multiple valvular involvement and increases with the severity of mitral regurgitation, indicating that RDW\% is also associated with inflammation severity for this subgroup of patients. A high RDW\% even after treatment may predict future stenotic valvular lesions in patients with ARC. Increase RDW\% might be due to high level of cytokines in circulation. From another perspective, these cytokine levels might be elevated in patients with rheumatic carditis even after appropriate medical treatment [30].

Another study has speculated that inflammation could suppress the maturation of red blood cells while shortening their lifespan, resulting in elevated RDW\% [31].

The RDW\% correlated positively with LVESD and PAP. While RDW\% correlated negatively with EF\% in cases with RHD.

Wasilewski et al. evaluated the prognostic value of RDW\% in patients with left ventricular systolic dysfunction, and found that the highest RDW\% tertile, with significantly lower LVEF\%, higher end-systolic and end-diastolic volumes as well as diameters, with a significantly higher occurrence of both mitral and aortic severe valve disease compared with patients in low and medium tertiles [32].

Anisocytosis may play a direct role in the onset and progressive worsening of HF. The erythrocyte size heterogeneity mirrors a reduced (often severely impaired) function of this essential corpuscular blood elements. In conditions of high anisocytosis, RBCs are often characterized by lower deformability and decreased oxygen-carrier capacity, thus contributing to reduced oxygenation of many peripheral tissues and cells (including cardiomyocytes), whilst abnormal erythrocytes may also actively participate in the pathogenesis of cardiac fibrosis through promotion or amplification of inflammation, cardiomyocyte stress and apoptosis [33].

As regard mean platelets count our results showed that, significant increase of mean value of platelets count in all cases with RHD compared to controls.

Cases with RHD in the presence of HF showed significant increase of mean platelets count in RHD subgroups either with one valve or more than one valve lesion.

Our study showed that positive correlation between platelets count with LVEDD and LVESD. Whlie negative correlation with EF\% in RHD cases.

Kucuk et al. mentioned that platelets count significantly higher in patient with acute rheumatic carditis compared with controls at the time of diagnosis, prior to the onset of treatment [30]. Platelets increase in number with stimulus such as inflammation and chronic systemic infection, and lead to over production of inflammatory cytokines [34].

In patients with ongoing inflammation, the increasing concentration of proin- 
flammatory cytokines, mainly IL-6, can lead to platelets release. This is associated with the stimulation of thrombopoietin generation by IL- 6 and with a direct effect of this cytokine on megakaryocytes. IL-6 causes an increase in the ploidy of megakaryocytic nuclei and an increase in cytoplasm volume, which in consequence leads to the production of a large number of blood platelets [35].

The mean value of PDW decreases in all cases with RHD compared to controls. In addition, as regard effect of HF in cases with RHD; there was significant decrease of MPV and PDW values in all cases with decompensated RHD compared to cases with compensated RHD. So, the heart failure has an effective role.

The mean values of MPV and PDW decreased in cases with decompensated RHD either they have one valve or more than one valve lesion compared to compensated cases.

The present study results showed negative correlation between MPV and PDW with LVEDD, LVESD, and PAP. While there was positive correlation with EF\% in cases with RHD.

In agreement with our results Sert et al., showed that increasing platelets count and decreasing MPV values in patients with rheumatic fever (RF) during the acute phase of illness [36].

Also Çilsal et al. mentioned that the carditis group had significantly lower MPV value than controls groups [37]. It has been hypothesized that decreased MPV values may indicate the intensity of the inflammatory process in conditions with elevated inflammatory markers. Excessive production of cytokines, such as IL- 6 and acute phase reactants, may affect the platelets production and suppress the size of the platelets released from the bone marrow. Moreover, IL-6 release and/or intensive consumption of larger platelets in the areas of inflammation may contribute to the low MPV during acute ARF attacks [38]. Further evidence to support this suggestion is that the inflammatory markers (ESR and CRP) were significantly elevated in cases with decompensated RHD. In contrast, Özdemir et al. [28] were unable to detect a significant alteration in MPV values in children with acute rheumatic carditis.

As well as Aşık et al. evaluated platelet indices and neutrophil/lymphocytic ratio(NLR) together in children with acute rheumatic fever, and found that significantly lower MPV and higher NLR values were found in patients with ARF compared to the control group were detected [39].

Our study showed that, increase CRP and ESR in all cases with decompensated RHD compared to cases with compensated RHD.

As regard RHD subgroups, our study showed increase CRP and ESR in cases with decompensated RHD either in cases they have one valve or in cases with more than one valve lesion compared to compensated RHD cases.

In RHD the present study showed that positive correlation between CRP and LVESD. While there is a negative correlation between CRP with EF\%. Also our study showed that positive correlation between ESR with LVEDD, LVESD and PAP. While negative correlation between ESR with EF\%. 
Çelik and Çelik found that, CRP and ESR, were significantly higher in RHD patients with acute carditis compared with the controls [40].

In an Egyptian study done by Gomaa et al. [41] found that the 1st-h ESR and $\mathrm{CRP}$, there were significantly increased values in rheumatic fever group as compared to controls. Kumar, [42] and Farghaly et al. [43] agreed with these results. These elevations may be explained by the ongoing inflammatory nature of the disease.

Comparison of the results in cases with CHD or RHD showed that, increase RDW\% in all cases with CHD compared to cases with RHD, as well as RDW\% value increase in all cases with decompensated CHD compared to cases with decompensated RHD.

Huang et al. evaluated the prognostic value of RDW\% for patients with $\mathrm{HF}$ and found that RDW\% is an effective index for HF prognosis evaluation. This means RDW\% should be measured when comprehensively assessing the prognosis of HF patients, and more intensive treatment for HF may be needed for patients with a higher RDW\% [44].

Also our study showed that, increase platelets count in all cases with RHD compared to cases with $\mathrm{CHD}$, also showed that increase platelets count in all cases with decompensated RHD compared to cases with decompensated CHD and this related to natural immune role of platelets, which elevated in acute phase reaction during the inflammatory process and can reflect the bone marrow cells activation secondary to stimulant effect of IL-6 [45].

In the presence of HF, the mean values of MPV and PDW showed lower values in all cases with RHD compared to cases with CHD.

Platelets activation has a very important role in inflammation; it has been observed to secrete mediators such as chemokines and cytokines [46]. The MPV that is associated with serious inflammation [47]. In previously reported studies, MPV values were shown to be significantly lower in rheumatoid arthritis and inflammatory bowel disease patients with active disease, compared to controls [48] [49].

Çelik and Çelik discovered increasing platelets count and decreasing MPV values in patients with acute rheumatic carditis (ARC), which reflect the inverse relationship between changes in platelets count and size [40]. The mechanism of increase in platelet volume is thought to be that inflammatory cytokines stimulate the production of large, reactive platelets, which have a shorter life span [50].

The mean value of CRP and ESR increase in all cases with RHD compared to cases with $\mathrm{CHD}$ also increase in cases with decompensated RHD than cases with decompensated CHD.

The best cut off point calculated in our study for RDW\% which is more than $13.8 \%$ with sensitivity of $51.4 \%$ and specificity of $57.5 \%$, at area under the curve (AUC) of $54.2 \%$, positive predictive value (PPV) is $77 \%$ and negative predictive value (NPV) is $48.2 \%$ as predictor for severity within congenital and rheumatic 
heart disease cases.

Our results agreed with Kucuk et al. found that RDW\% value above $13.3 \%$ can detect the inflammation significantly $(\mathrm{P}=0.004)$, with $54 \%$ sensitivity and $68.4 \%$ specificity at AUC of 0.61 (95\% CI: 54 - 69.5) with significant positive correlation between the RDW\% values before the treatment and the severity of mitral regurgitation. Moreover in regression analysis, RDW\% was independent predictor for severe mitral regurgitation [31].

Also the best cut-off point regarding platelets count was less than $295 \times 10^{3} / \mu \mathrm{l}$ with sensitivity of $61.3 \%$, specificity of $92.5 \%$, AUC $74.2 \%$, PPV of $95.8 \%$ and NPV of $46.3 \%$.

In addition, the best cut-off point for MPVfl was more than $8.7 \mathrm{fl}$ with sensitivity of $47.7 \%$, specificity of $82.5 \%$ at AUC $59.7 \%$, PPV is $88.3 \%$ and NPV is $36.3 \%$. also the best cut of point regarding PDWfl was found more than $13.5 \mathrm{fl}$ with sensitivity is $36 \%$, specificity of $87.5 \%$ at AUC $58.9 \%$, PPV $88.9 \%$ and NPV is $33 \%$.

The larger platelets are more active than the smaller ones. This leads to the production of more thromboxane A2 and beta thromboglobulin and increases the propensity to the prothrombotic state [51]. Previous study have shown that increased MPV increases the risk of atherothrombosis and thus cardiovascular events [52].

In Martin Garcia et al. found that thrombocytopenia significantly increased the risk of mortality in Eisenmenger syndrome (ES). Furthermore, raised MPV, severe secondary erythrocytosis and anaemia, but not platelets count were associated with an increased risk of thrombotic events among ES patients [53].

\section{Conclusion}

The mean values of RDW\% and platelets count were significantly higher in children with either congenital or rheumatic heart disease especially those with decompensated cardiac function and those with multiple valve lesion. Mean platelets count was decreased in children with cyanotic CHD (either compensated or decompensated) in comparison to those with acyanotic CHD. The MPV and PDW were increased in children with decompensated CHD but decreased in children with decompensated RHD. So they could be considered as simple markers in the follow up of patients with CHD or RHD for early detection of serious complication.

\section{Conflicts of Interest}

The authors declare no conflicts of interest regarding the publication of this paper.

\section{References}

[1] Liu, Y., Chen, S., Zuhlke, L., Black, G.C, Choy, M., Li, N., et al. (2019) Global Birth Prevalence of Congenital Heart Defects 1970-2017: Updated Systematic Review and Meta-Analysis of 260 Studies. International Journal of Epidemiology, 48, 455-463. https://doi.org/10.1093/ije/dyz009 
[2] Bouma, B.J. and Mulder, B.J. (2017) Changing Landscape of Congenital Heart Disease. Circulation Research, 120, 908-922. https://doi.org/10.1161/CIRCRESAHA.116.309302

[3] Woldu, B. and Bloomfield, G.S. (2016) Rheumatic Heart Disease in the TwentyFirst Century. Current Cardiology Reports, 18, Article No. 96. https://doi.org/10.1007/s11886-016-0773-2

[4] Watkins, D.A., Johnson, C.O., Colquhoun, S.M., Karthikeyan, G., Beaton, A., Bukhman, G., et al. (2017) Global, Regional, and National Burden of Rheumatic Heart Disease, 1990-2015. New England Journal of Medicine, 377, 713-722. https://doi.org/10.1056/NEJMoa1603693

[5] Qin, Y., Wang, P., Huang, Z., Huang, G., Tang, J., Guo, Y., et al. (2017) The Value of Red Cell Distribution Width in Patients with Ovarian Cancer. Medicine, 96, e6752.https://doi.org/10.1097/MD.0000000000006752

[6] Kojima, T., Yasuhara, J., Kumamoto, T., Shimizu, H., Yoshiba, S., et al. (2015) Usefulness of the Red Blood Cell Distribution Width to Predict Heart Failure in $\mathrm{Pa}$ tients with a Fontan Circulation. American Journal of Cardiology, 116, 965-968. https://doi.org/10.1016/j.amjcard.2015.06.020

[7] Gremmel, T., Ay, C., Riedl, J., Kopp, C.W., Eichelberger, B., Koppensteiner, R., et al. (2016) Platelet-Specific Markers Are Associated with Monocyte-Platelet Aggregate Formation and Thrombin Generation Potential in Advanced Atherosclerosis. Thrombosis and Haemostasis, 115, 615-6621.https://doi.org/10.1160/th15-07-0598

[8] Mirzaie, A.Z., Abolhasani, M., Ahmadinejad, B. and Panahi, M. (2012) Platelet Count and MPV, Routinely Measured but Ignored Parameters Used in Conjunction with the Diagnosis of Acute Coronary Syndrome: Single Study Center in Iranian Population, 2010. Medical Journal of the Islamic Republic of Iran, 26, 17-21.

[9] Massin, M.M. (2012) Relation between Red Cell Distribution Width and Clinical Outcome after Surgery for Congenital Heart Disease in Children. Pediatric Cardiology, 33, 1021-1025.https://doi.org/10.1007/s00246-012-0220-0

[10] Polat, V., Iscan, S., Etli, M., El Kılıc, H., Gürsu, Ö., Eker, E., et al. (2014) Red Cell Distribution Width as a Prognostic Indicator in Pediatric Heart Disease and after Surgery. BioMed Research International, 2014, Article ID: 681679. https://doi.org/10.1155/2014/681679

[11] Mawlana, W, Donia, A. and Elamrousy, D. (2014) Relation between Red Cell Distribution Width and Left Ventricular Function in Children with Heart Failure. International Scholarly Research Notices, 2014, Article ID: 234835. https://doi.org/10.1155/2014/234835

[12] Semba, R.D., Patel, K.V., Ferrucci, L., Sun, K., Roy, C.N., Guralnik, J.M., et al. (2010) Serum Antioxidants and Inflammation Predict Red Cell Distribution Width in Older Women: The Women's Health and Aging Study I. Clinical Nutrition, 29, 600-604. https://doi.org/10.1016/j.clnu.2010.03.001

[13] Animasahun, B.A., Itiola, J. and Adekunle, MO. (2020) Comparison of Erythrocyte Indices and Haematological Indices as Markers of Iron Status of Lagos Children with Cyanotic Congenital Heart Disease. Annals of Blood, 5, 1-12. http://dx.doi.org/10.21037/aob.2020.01.01

[14] Aulakh, R., Sohi, I., Singh, T. and Kakkar, N. (2009) Red Cell Distribution Width (RDW) in the Diagnosis of Iron Deficiency with Microcytic Hypochromic Anemia. The Indian Journal of Pediatrics, 76, 265-268. https://doi.org/10.1007/s12098-009-0014-4

[15] Yudha, A.P., Widjaja, S. and Salimo, H. (2017) Relationship between The Value of 
Red Cell Distribution Width and Left Ventricular Function in Children with Acyanotic Congenital Heart Disease Patients. Sari Pediatri, 18, 339-344.

[16] Yang, H.J., Liu, X., Qu, C., Shi, S.-B., Liang, J.-J. and Yang, B. (2018) Usefulness of Red Blood Cell Distribution Width to Predict Heart Failure Hospitalization in Patients with Hypertrophic Cardiomyopathy. International Heart Journal, 59, 779-785. https://doi.org/10.1536/ihj.17-507

[17] Al-Najjar, Y., Goode, K.M., Zhang, J., Cleland, J.G.F. and Clark, A.L. (2009) Red Cell Distribution Width: An Inexpensive and Powerful Prognostic Marker in Heart Failure. European Journal of Heart Failure, 11, 1155-1162. https://doi.org/10.1093/eurjhf/hfp147

[18] Park, T.S. and Zambidis, E.T. (2009) A Role for the Renin-Angiotensin System in Hematopoiesis. Haematologica, 94, 745-747.

https://doi.org/10.3324/haematol.2009.006965

[19] Lill, M.C., Perloff, J.K. and Child, J.S. (2006) Pathogenesis of Thrombocytopenia in Cyanotic Congenital Heart Disease. The American Journal of Cardiology, 98, 254258. https://doi.org/10.1016/j.amjcard.2006.01.083

[20] Mese, T., Guven, B., Yilmazer, M.M., Karadeniz, C., Ozdemir, R. and Doksoz, O. (2018) Platelet Activation Markers in Children with Congenital Heart Disease Associated with Pulmonary Arterial Hypertension. Congenital Heart Disease, 13, 506-511. https://doi.org/10.1111/chd.12616

[21] Kaya, M.G., Elcik, D., Akpek, M., Kelesoglu, S., Sahin, O., Sarli, B. and Lam, Y.Y. (2014) Mean Platelet Volume Levels Predict Pulmonary Artery Hypertension in Patients with Atrial Septal Defect. Acta Cardiologica, 69, 161-166. https://doi.org/10.1080/AC.69.2.3017297

[22] Sato, M., Inai, K., Ogiso, M., Kudo, Y., Nishimura, T., Mori, H., et al. (2020) Platelet Volume Indices Correlate to Severity of Heart Failure and Have Prognostic Value for both Cardiac and Thrombotic Events in Patients with Congenital Heart Disease. European Heart Journal, 41, ehaa946.2208. https://doi.org/10.1093/ehjci/ehaa946.2208

[23] Berger, G., Azzam, Z.S., Hoffman, R. and Yigla, M. (2009) Coagulation and Anticoagulation in Pulmonary Arterial Hypertension. Israel Medical Association Journal, 11, 376-379.

[24] Varol, E., Uysal, B.A., Ozaydin, M., Ozaydin, M., Erdogan, D. and Dogan, A. (2011) Platelet Indices in Patients with Pulmonary Arterial Hypertension. Clinical and Applied Thrombosis/ Hemostasis, 17, E171-E174. https://doi.org/10.1177\%2F1076029610379400

[25] Açikgöz, N., Ermiş,N., Yağmur, J., Cansel, M., Ataş, H., Pekdemir, H. and Ozdemir, R. (2011) The Evaluation of Mean Platelet Volume Levels in Patients with Idiopathic and Ischemic Cardiomyopathy: An Observational Study. Anadolu Kardiyoloji Dergisi, 11, 595-599. https://doi.org/10.5152/akd.2011.161

[26] Nassef, Y.E., Hamed, M.A. and Aly, H.F. (2014) Inflammatory Cytokines, Apoptotic, Tissue Injury and Remodeling Biomarkers in Children with Congenital Heart Disease. Indian Journal of Clinical Biochemistry, 29, 145-149. https://doi.org/10.1007/s12291-013-0341-0

[27] Kantor, P.F. and Rusconi, P. (2011) Biomarkers in Pediatric Heart Failure: Their Role in Diagnosis and Evaluating Disease Progression. Progress in Pediatric Cardiology, 31, 53-57. https://doi.org/10.1016/j.ppedcard.2010.11.012

[28] Ozdemir, R., Karadeniz, C., Doksoz, O., Celegen, M., Yozgat, Y., Guven, B., et al. (2014) Are Mean Platelet Volume and Platelet Distribution Width Useful Parame- 
ters in Children with Acute Rheumatic Carditis? Pediatric Cardiology, 35, 53-56. https://doi.org/10.1007/s00246-013-0738-9

[29] Karpuz, D., Giray, D., Ozyurt, A., Bozlu, G., Unal, S. and Hallioglu, O. (2017) Can Whole-Blood Parameters Be Used in Follow-Up of Children with Rheumatic Valvular Heart Disease? Cardiology in the Young, 27, 764-769.

https://doi.org/10.1017/S1047951116001281

[30] Kucuk, M., Ozdemir, R., Karadeniz, C., Celegen, K., Demirol, M., Yilmazer, M.M., et al. (2016) Red Blood Cell Distribution Width: Can It Be a Predictive Marker for Long-Term Valvular Involvement in Children with Acute Rheumatic Carditis? International Journal of Laboratory Hematology, 38, 569-575. https://doi.org/10.1111/ijlh.12544

[31] Jo, Y.H., Kim, K., Lee, J.H., Kang, C.W., Kim, T.G., Park, H.-M., et al. (2013) Red Cell Distribution Width Is a Prognostic Factor in Severe Sepsis and Septic Shock. The American Journal of Emergency Medicine, 31, 545-548. https://doi.org/10.1016/j.ajem.2012.10.017

[32] Wasilewski, J., Pyka, Ł., Hawranek, M., Tajstra, M., Skrzypek, M., Wasiak, M., et al. (2018) Prognostic Value of Red Blood Cell Distribution Width in Patients with Left Ventricular Systolic Dysfunction: Insights from the COMMIT-HF Registry. Cardiology Journal, 25, 377-385. https://doi.org/10.5603/CJ.a2017.0037

[33] Salvagno, G.L., Sanchis-Gomar, F., Picanza, A. and Lippi, G. (2015) Red Blood Cell Distribution Width: A Simple Parameter with Multiple Clinical Applications. Critical Reviews in Clinical Laboratory Sciences, 52, 86-105. https://doi.org/10.3109/10408363.2014.992064

[34] Wæhre, T., Damås, J.K., Yndestad, A., Taskén, K., Pedersen, T.M., Smith, C., et al. (2004) Effect of Activated Platelets on Expression of Cytokinesin Peripheral Blood Mononuclear Cells-Potential Role of Prostaglandin E2. Thrombosis and Haemostasis, 92, 1358-1367. https://doi.org/10.1160/TH04-03-0146

[35] Senchenkova, E., Komoto, S., Russell, J., Almeida-Paula, L.D., Yan, L.-S. and Zhang, S.L. (2013) Interleukin-6 Mediates the Platelet Abnormalities and Thrombogenesis Associated with Experimental Colitis. American Journal of Pathology, 183, 173-181. https://doi.org/10.1016/j.ajpath.2013.03.014

[36] Sert, A., Aypar, E. and Odabas, D. (2013) Mean Platelet Volume in Acute Rheumatic Fever. Platelets, 24, 378-382. https://doi.org/10.3109/09537104.2012.701029

[37] Çilsal, E., Pektaş, A., Pektaş, B.M. and Koca, B.H. (2018) Echocardiographic and Laboratory Findings of Turkish Children during the First Attack of Acute Rheumatic Fever. European Journal of Therapeutics, 24, 77-82. https://doi.org/10.5152/Eur]Ther.2017.191

[38] Gruys, E., Toussaint, M.J., Niewold, T.A. and Koopmans, S.J. (2005) Acute Phase Reaction and Acute Phase Proteins. Journal of Zhejiang University-SCIENCE B, 6, 1045-1056.

[39] Aşık, A., Selçuk Duru, N. and Elevli, M. (2019) An Evaluation of Platelet Parameters and Neutrophil/Lymphocyte Ratios in Children with Acute Rheumatic Fever. Pediatric Research, 6, 37-43. https://doi.org/10.4274/jpr.galenos.2018.30075

[40] Çelik, S.F. and Çelik, E. (2018) The Neutrophil-to-Lymphocyte Ratio and Mean Platelet Volume Can Be Associated with Severity of Valvular Involvement in $\mathrm{Pa}$ tients with Acute Rheumatic Carditis. Cardiovascular Journal of Africa, 29, 296-300. https://doi.org/10.5830/CVJA-2018-031

[41] Gomaa, M.H., Ali, S.S., Fattouh, A.M., Hamza, H.S. and Badr, M.M. (2018) MBL2 Gene Polymorphism rs 1800450 and Rheumatic Fever with and without Rheumatic 
Heart Disease: An Egyptian Pilot Study. Pediatric Rheumatology, 16, Article No. 24. https://doi.org/10.1186/s12969-018-0245-x

[42] Kumar, R. (2016) Spectrum of Clinical Presentation of Rheumatic Fever and Rheumatic Heart Disease Patients. Annals of International Medical and Dental Research, 2, 49-51.

[43] Farghaly, H.S., Gad, E.F., Hassan, A.-E.A, Tahr Asherif and Abdou, M.A.A. (2017) Evaluation of Adrenomedullin Levels in Children with Acute Rheumatic Fever and Its Correlation to Left Ventricular Function. American Journal of Medicine and Medical Sciences, 7, 74-78.

[44] Huang, Y.L., Hu, Z.D., Liu, S.J., Sun, Y., Qin, Q., Qin, B.-D., et al. (2014) Prognostic Value of Red Blood Cell Distribution width for Patients with Heart Failure: A Systematic Review and Meta-Analysis of Cohort Studies. PLOS ONE, 9, e104861. https://doi.org/10.1371/journal.pone.0104861

[45] Unsal, E., Aksaray, S., Koksal, D. and Sipit, T. (2005) Potential Role of Interleukin 6 in Reactive Thrombocytosis and Acute Phase Response in Pulmonary Tuberculosis. Postgraduate Medical Journal, 81, 604-607. http://dx.doi.org/10.1136/pgmj.2004.030544

[46] Bath, P. and Butterworth, R. (1996) Platelet Size: Measurement, Physiology and Vascular Disease. Blood Coagulation \& Fibrinolysis, 7, 157-161. https://doi.org/10.1097/00001721-199603000-00011

[47] Pitchford, S. and Page C. (2006) Platelet Activation in Asthma: Integral to the Inflammatory Response. Clinical \& Experimental Allergy, 36, 399-401. https://doi.org/10.1111/j.1365-2222.2006.02462.x

[48] Gasparyan, A.Y., Stavropoulos-Kalinoglou, A., Toms, T.E., Douglas, K.M.J. and Kitas, G.D. (2010) Association of Mean Platelet Volume with Hypertension in Rheumatoid Arthritis. Inflammation \& Allergy-Drug Targets, 9, 45-50. https://doi.org/10.2174/187152810791292854

[49] Kapsoritakis, A.N., Koukourakis, M.I., Sfiridaki, A., Potamianos, S.P., Kosmadaki, M.G., Koutroubakis, I.E., et al. (2001) Mean Platelet Volume: A Useful Marker of Inflammatory Bowel Disease Activity. American Journal of Gastroenterology, 96, 776-781.

[50] Endler, G., Klimesch, A., Sunder-Plassmann, H., Schillinger, M., Exner, M., Mannhalter, C., et al. (2002) Mean Platelet Volume Is An Independent Risk Factor for Myocardial Infarction but Not for Coronary Artery Disease. British Journal of Haematology, 117, 399-404. https://doi.org/10.1046/j.1365-2141.2002.03441.x

[51] Hasan-Ali, H. and Mosad, E. (2015) Changes in Platelet, Coagulation, and Fibrinolytic Activities in Mitral Stenosis after Percutaneous Mitral Valvotomy: Role of Hemodynamic Changes and Systemic Inflammation. Clinical and Applied Thrombosis/ Hemostasis, 21, 339-347. https://doi.org/10.1177\%2F1076029614533144

[52] Chu, S.G., Becker, R.C., Berger, P.B., Bhatt, D.L., Eikelboom, J.W., Konkle, B., et al. (2010) Mean Platelet Volume as a Predictor of Cardiovascular Risk: A Systematic Review and Meta-Analysis. Journal of Thrombosis and Haemostasis, 8, 148-156. https://doi.org/10.1111/j.1538-7836.2009.03584.x

[53] Martin Garcia, A.C., Arachchillage, D.R.J., Kempny, A., Alonso-Gonzalez, R., Martin-Garcia, A., Uebing, A., et al. (2017) Low Platelet Count and Its Relation to Death in Adults with Eisenmenger Syndrome. European Heart Journal, 38, ehx504.P4533. https://doi.org/10.1093/eurheartj/ehx504.P4533 